\title{
Pediatric Dental Sleep Medicine: the ADA Children's Airway Conference
}

\author{
Rose D. Sheats, DMD, MPH, Associate Editor, Journal of Dental Sleep Medicine
}

The American Dental Association (ADA) is to be commended for its August 2018 inaugural conference on "Children's Airway Health - A Practical Conference". The 2-day conference was timely, well-organized, and included a broad range of topics presented by representatives from a number of disciplines, including sleep medicine, otolaryngology, surgery, pediatric dentistry, orthodontics, psychology, orofacial myology, and business.

The objectives of the ADA conference were ambitious and included allowing speakers to present various treatment options with only anecdotal evidence to support success of treatment. The demand for guidance in managing pediatric sleep-disordered breathing (SDB) is indisputable, but the conference failed to recognize some glaring omissions that must be considered before any definitive recommendations are made and implemented. These factors include but are not limited to the following questions:

1. What are the appropriate criteria to define a pediatric dental sleep medicine patient? The medicolegal definition is birth until 18 years of age. Is this an appropriate definition to be applied to dental sleep medicine?

2. What are the diagnostic criteria and who is the appropriate clinician for the diagnosis of SDB in children? Are snoring, mouth breathing, or behavioral issues enough?

3. What is the dentist's role in screening children for SDB?

4. What are the appropriate screening protocols based on evidence and what known risk factors should be used?

5. What is the best age for intervention, or is age the appropriate indicator?

6. What treatment options are available that have scientific basis?

7. What are the anticipated side effects of the proposed treatments and can they be managed?

8. Who is responsible for coordination of multiple aspects of pediatric patients with SDB?

9. What is the definition of treatment success?

10. What follow-up protocols exist?
Although it is laudable that the ADA conference aspired to equip dentists with tools and the confidence to begin treating pediatric patients for SDB upon return to their practices "Monday morning", it may have been premature to endorse treatment options before clear guidelines have been developed to describe the entire clinical pathway of dental management of a pediatric dental sleep medicine (DSM) patient.

Furthermore, to bolster the value of such treatment possibilities, they should be evaluated in a nonbiased controlled manner to ascertain their benefit. If found effective, recommendations on the most appropriate candidates for such treatment would enhance their value. Approaching treatments for pediatric sleep disorders in any other way would be a departure from the norm, creating unknown consequences for the patient, his or her family, and the dentists. We must resist a rush to solve a problem without a sound plan for doing so.

As is common for groundbreaking conferences such as these, more questions are raised than solutions presented. Pediatric SDB is a complex field. One of the ADA's stated objectives of the conference was to enable attendees to "[i]mmediately treat children falling within their scope of expertise." Dental treatment of children in whom SDB has been diagnosed should not be undertaken without sufficient education and training, not simply by attending a 2-day conference on the topic, and must be based on a rational clinical pathway that has been developed after careful consideration of the medical history, family history, and objective assessment as well as all available medical evidence supporting appropriate treatment.

Parents and clinicians are seeking treatment options to manage conditions thought to be associated with pediatric SDB, such as childhood bruxism, snoring, secondary sleep enuresis, and behavioral issues. They have lost patience with pediatricians, allergists, otolaryngologists, orthodontists, and sleep physicians who intone that their child is normal, and/or will "outgrow" the problem, or is too young to treat.

The ADA has demonstrated leadership in pediatric dental sleep medicine by organizing this seminal conference. I look forward to continued advancement of the field and hope that the ADA conference will inspire our 
dental leaders to arrive at a consensus about how best to treat the pediatric patient in whom SDB has been diagnosed. The ADA has another conference scheduled for March 2019, and the manner in which it acknowledges gaps in our knowledge of pediatric dental sleep medicine and its plan to address them will establish what role it may play in the evolution of the field.

\section{CITATION}

Sheats, RD. Pediatric Dental Sleep Medicine: The ADA Children's Airway Conference. Journal of Dental Sleep Medicine. 2018;5(4):107-108.

\section{REFERENCES}

1. Children's Airway Health - A Practical Conference. ADA CE Live. https://www.ada.org/en/education-careers/continuing-education/adace-live?utm_medium=VanityUrl. Accessed 9.10.18.

\section{SUBMISSION AND CORRESPONDENCE INFORMATION}

Submitted in final revised form September 11, 2018 Accepted for publication September 11, 2018

Address correspondence to: Rose Sheats, DMD, MPH, University of North Carolina, Chapel Hill, NC; Telephone: (919) 537-8003; Email: rose_sheats@unc.edu.

\section{DISCLOSURE STATEMENT}

Dr. Sheats is an editor of the Journal of Dental Sleep Medicine. Dr. Sheats is also a member of the American Academy of Dental Sleep Medicine Board of Directors. Her opinion does not necessarily constitute the opinion of the AADSM board. 